\title{
DESIGN, CONSTRUCTION, AND OPERATION OF SMALL COSMIC RAYS DETECTORS AT UNIVERSIDAD DE GUANAJUATO, MEXICO
}

\author{
Julian Felix ${ }^{* \dagger}$ \\ Author affiliation \\ Laboratorio de Partículas Elementales, Departamento de Física. DCeI, CL. Universidad de \\ Guanajuato E-mail: felixafisica.ugto.mx
}

\begin{abstract}
Since 30 years ago Universidad de Guanajuato students and professors have participated in many international experimental high Energy physics collaborations, working at Fermilab USA, mainly [1]. Slow progress, and a tendency to impoverish, has observed in the development of this area of science in Mexico. We have disregarded the scientific and technological development of Mexico, preparing students, and collaborating, outside. Graduate students do not contribute to the economical-technological development of Mexico, and private industry does not participate. To remediate this situation and improve the level of science and technology in Mexico, we have created the Laboratory for elementary particles (laboratorio de partículas elementales [2]). Here students of the three levels -BSc, Master, and $\mathrm{PhD}$ in physics- design, construct, calibrate and operate small cosmic rays detectors. Some local equipment vendors, and small enterprises, participate. We consider that this new way of proceeding will benefit the scientific relations with international collaborations, improve scientific and technological preparation of students, force the participation of local industry, and contribute to the economic development of Mexico. We present this strategy, some physical results obtained with the constructed equipment, and some conclusions.
\end{abstract}

38th International Conference on High Energy Physics

3-10 August 2016

Chicago, USA

\footnotetext{
* Speaker.

$\dagger$ To the many students who have participated in this project. Francisco Javier Rosas Torres, Jazmín Sánchez Sánchez, MiguelÁngel Hernández Morquecho, Karla Natalia Herrera Guzmán, Raúl Alejandro Gutiérrez Sánchez, Oscar Eduardo Moreno Palacios, Everardo Granados Vázquez, Jordán de Jesús Ortiz Villanueva, Gerardo de Jesús Rodríguez Becerra, Carlos Andrés Cervantes Vera, Diego Armando Andrade Aldana, Alfredo Martínez Vega, Luis Jorge Arceo Miquel, Aurora Cecilia Araujo Martínez. To CoNaCyT Grant 223179, CB-2013-01, Fondo SEP CONACYT.
} 


\section{Introduction}

Since 30 years ago Universidad de Guanajuato students and professors have participated in many international experimental high Energy physics collaborations, working at Fermilab USA, mainly [1], this is inside the tradition started by Leon M. Lederman in the late 1970 in Latinamerica. Figure 1. Up to these days, there are many scientific groups in experimental high energy physics working in many institutions of Mexico. Laboratorio de Partículas Elementales, Universidad de Guanajuato; Universidad Autónoma de San Luis Potosí; Universidad Michoacana de San Nicolás de Hidalgo; CINVESTAV-

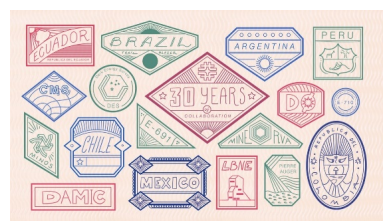

Figure 1: Latinamerica at Fermilab. From Symmetry.

Mérida; Universidad de Chiapas; UNAM; CINVESTAV; IBERO;

Benemérita Universidad Autónoma de Puebla; Universidad de Guadalajara; and Universidad Autónoma de Sinaloa. These groups collaborate with CERN (http://home.cern/) or with Fermilab (http://www.fnal.gov/). They really do not locally create technology. Slow progress, and a tendency to impoverish, has been observed in the development of this area of science in Mexico, for we do not generate our own projects in Mexico, we collaborate. We lack of a national laboratory. We have disregarded the scientific and technological development of Mexico, preparing students, and collaborating, outside. Locally, we have almost nothing. Graduate students do not contribute to the economical-technological development of Mexico, and private industry does not participate, for the above situation.

\section{The Laboratory for Elementary Particles (El Laboratorio de Partículas Elementales)}

To remediate this situation and improve the level of science and technology in Mexico, we have created the Laboratory for elementary particles (laboratorio de partículas elementales [2]). Here students of the three levels -BSc, Master, and PhD in physics- design, construct, calibrate and operate small cosmic rays detectors. Some local equipment vendors -computer, material, electronic equipment-, and small enterprises -electronic design and fabrication -, participate. The laboratory has three areas: Radiation Detectors -mechanics, electronics, calibration -, high performance computing, and accelerators. There

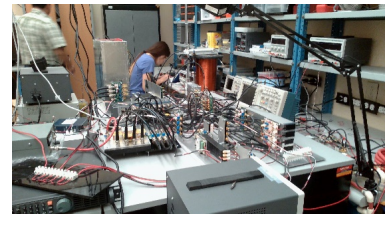

Figure 2: Laboratorio de partículas elementales. One view. are about nineteen associated students in this laboratory. 15 bachelor students, 1 master student, and three doctoral students. Figure 2, one view of laboratorio de partículas elementales.

\section{Design, Construction, and Run of Small Cosmic Rays Detectors}

There is a big project to create a 1 cubic meter detector of 10000 channels, based on different technologies. This is under planning and design. To accomplish this big detector, there have been many tests and studies. We have planned, design, constructed, and tested ten small cosmic rays detectors, as follows: one hybrid basic detector cell, with both Cerenkov and ionization detector 
channels, two of each detector channels; it works very well, see the presentation at this ICHEP 2016.

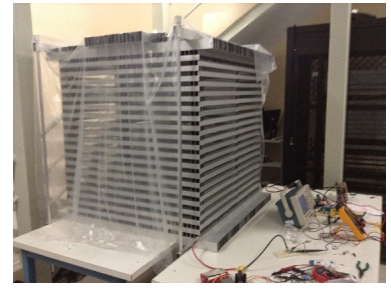

Figure 3: On construction 10000 channel cosmic ray detector.

One three Aluminum block cosmic rays detector, it runs very well, see the presentation at this ICHEP 2016. One $10 \mathrm{~cm} \mathrm{X} 10 \mathrm{~cm}$ $\mathrm{X} 1 \mathrm{~cm} 4$ glass channel detector. One four Aluminum channel detector. One four Polymaq channel detector. One three channel cosmic rays detector. One six electron gas channel detector. One four mini wire chamber detector. One three channel time of flight system. One 32 channel cosmic rays detector. All prototypes are designed to get analogical and digital signal.

This technology was planned, designed, constructed, tested, and run by the associated students to this laboratory of elementary particles. All the basic electronics was planned, designed, and created.

Figure 3-4, two different Cosmic ray detectors. There is some Electronics also. Students learn how to create, test and run technology for cosmic ray detection. Big detectors are created with the integration of many small detectors.

\section{Results}

There are some results obtained with these equipment. The technical training of 19 students, students are prepared to create technology based on basic science. Ten small prototypes for cosmic ray detectors. All detectors run very well. Figure 5, the display of all prototypes properly working at Fermilab. All equipment piece was tested for digital and analogical signal. Figure 6, some results. All equipment was characterized by the number of counts as function of applied high voltage. Figure 7, some results. All equipment was run for cosmic ray flux.

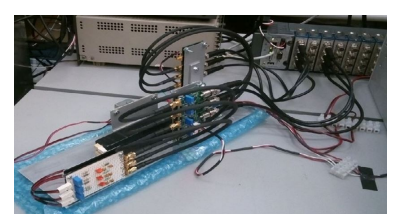

Figure 4: Six channel electron gas cosmic ray detector.

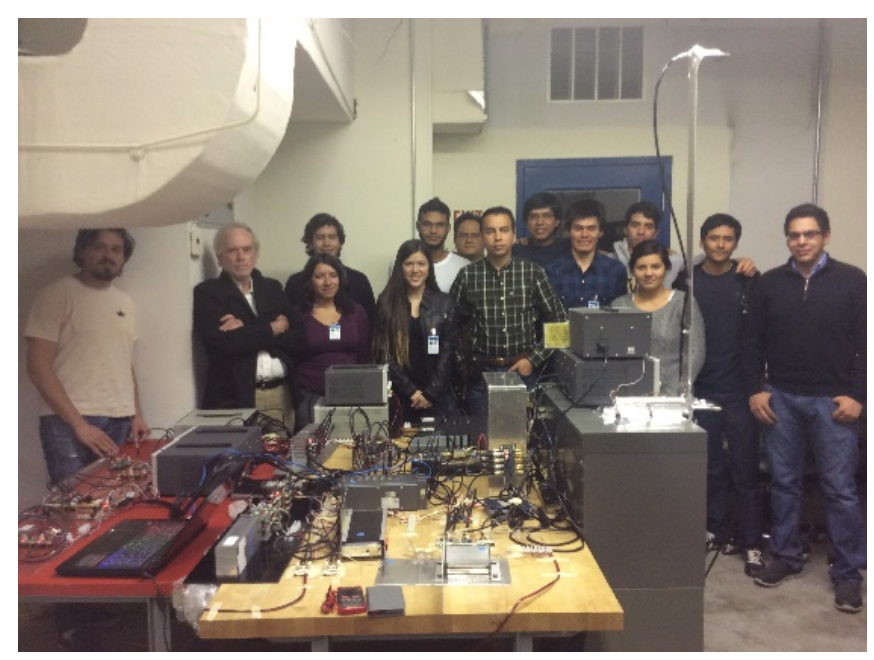

Figure 5: Equipment properly working at Fermilab, and students. 


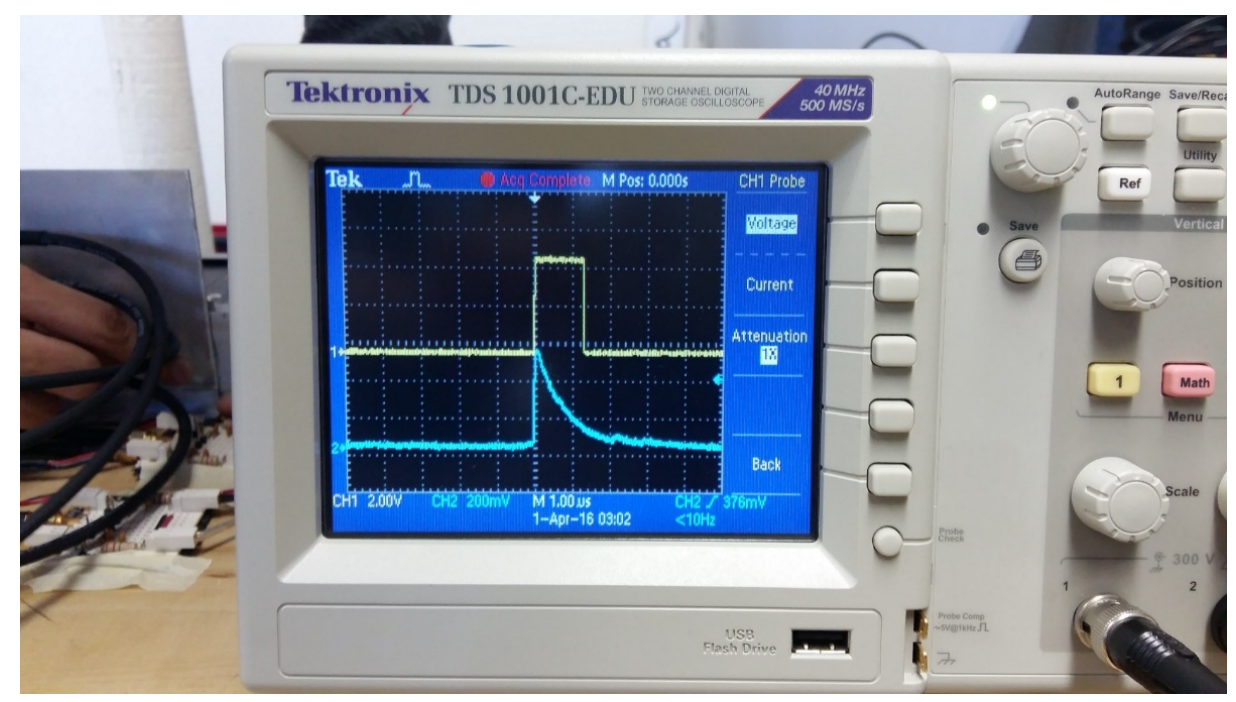

Figure 6: Typical analog and digital signals display.

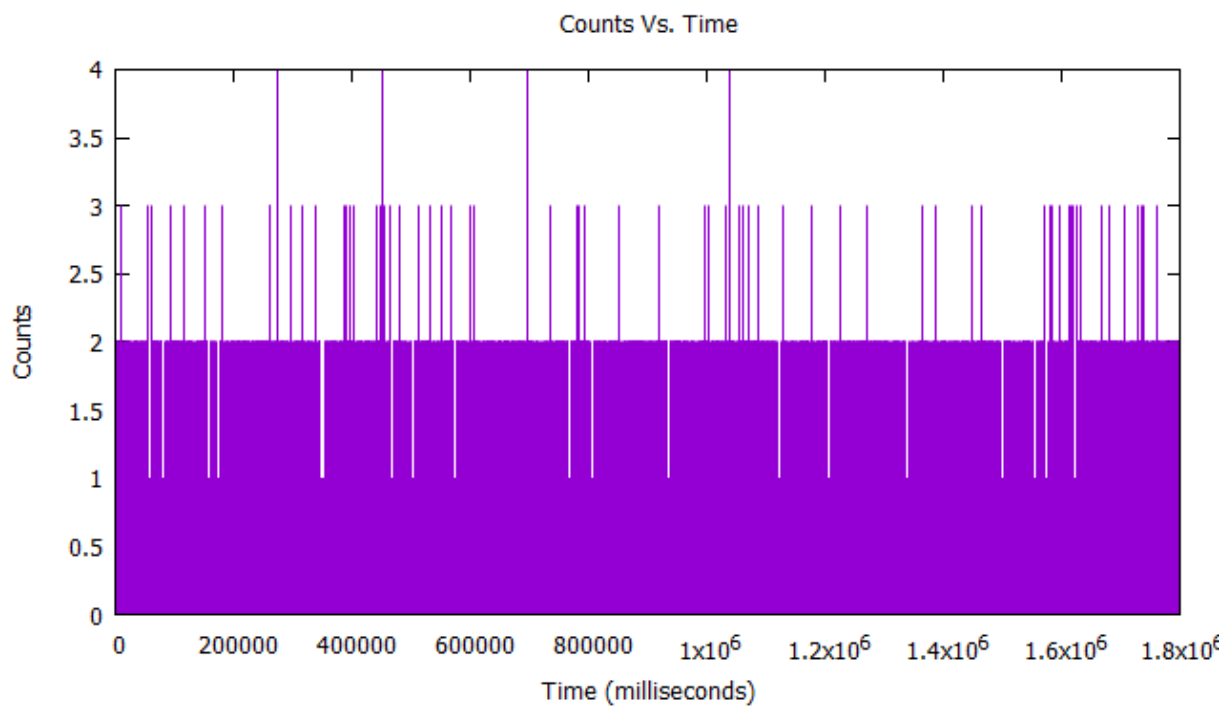

Figure 7: Typical data display, counts vs time.

\section{Conclusions}

We consider that this new way of proceeding -create local facilities and scientific training- will benefit the scientific relations with international collaborations, improve the scientific and technological preparation of students, force the participation of local industry, and contribute to the economic development of Mexico.

\section{References}

[1] http://www.symmetrymagazine.org/article/march-2014/30-years-of-inter-american-collaboration.

[2] http://laboratoriodeparticulaselementales.blogspot.mx. 